\title{
Electrochemically activated carbon fiber sensors as fast and sensitive adenine metabolite amperometric detectors
}

\author{
Kholoud Mohammed Abou El-Nour a,b \\ a Department of Chemistry, University of Florida, Gainesville, Florida, 32611-7200, USA \\ b Department of Chemistry, University of Suez Canal, Ismailia, 41522, Egypt \\ *Corresponding author at: Department of Chemistry, University of Suez Canal, Ismailia, 41522, Egypt. \\ Tel.: +20643208760; fax: +20643230416. E-mail address: kabolnoor@yahoo.com (K.M.A. El-Nour).
}

\section{ARTICLE INFORMATION}

Received: 18 February 2013

Received in revised form: 23 March 2013

Accepted: 25 March 2013

Online: 30 June 2013

\section{KEYWORDS}

Carbon fiber sensor

2,8-Dihydroxyadenine

Fast scan voltammetry

Electrochemical biosensors

Electrochemically activated sensor

Nanostructured ultramicroelectrode

\section{Introduction}

Adenine is a purine found in all tissues of the body. Adenine is converted to adenylate by the purine salvage enzyme adenine phosphoribosyltransferase (APRT), which converts adenine from the nucleotide pool into adenosine monophosphate in the presence of 5-phosphoribosyl-1pyrophosphate [1]. If adenine phosphoribosyltransferase is deficient, the accumulated adenine is either excreted in the urine or is converted by xanthine oxidase to the nephrotoxic 2,8-dihydroxyadenine (DHA) via the 8-hydroxyadenine intermediate [1].

Since 2,8-dihydroxyadenine is insoluble in water at physiological $\mathrm{pH}$ and at concentrations $>1-3 \mu \mathrm{g} / \mathrm{mL}$, it precipitates forming crystals [2]. The precipitation and crystallization of 2,8-DHA can lead to stone formation in various parts of the urinary tract [3], leading to the loss of the kidneys.

Various methods have been developed for the determination of 2,8-DHA in body fluids $[1,4,5]$. Investigations of the redox behavior of biologically occurring compounds by means of electrochemical techniques have the potential for providing valuable insights into biological redox reactions of these molecules. Due to their high sensitivity voltammetric methods were used here to study the redox behavior of 2,8DHA by means of fast scan cyclic voltammetry at a nanostructured carbon fiber sensor.

In this work, highly active carbon fiber ultramicro electrodes (CFEs) were produced, to act as sensors in sensitive determinations of 2,8-DHA under physiological conditions. The procedure that was adapted in our lab to activate the CFE surface for the determinations of 2,8-DHA was found to produce, a stable background current at the carbon fiber sensor

\begin{abstract}
A direct and fast voltammetric method of determination of 2,8-dihydroxyadenine (2,8-DHA) with a novel nanostructured carbon fiber sensor (CFS), is revealed in this work. To achieve electrochemical activation process generates a nanostructured carbon fiber surface, which is Balt solution (HBSS) : phosphate buffer $(1: 5) \mathrm{pH}=7.4$, with different scan rates of 政, 500 and $1000 \mathrm{~V} / \mathrm{s}$. The potential window in the determinations was from -1.0 to $1.5 \mathrm{~V}$ (vs.

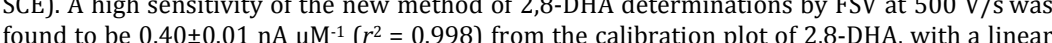
dynamic range from 1-20 $\mu \mathrm{M}$, and a limit of detection of $1 \mu \mathrm{M}$ under physiological conditions.
\end{abstract}

(CFS). The activation is based on the electrochemical pretreatment (ECP) of the CFS surface, by continuous cycling, for $30 \mathrm{~min}$, of the electrode potential, at a scan rate of $10 \mathrm{~V} / \mathrm{s}$ in a potential window from -1.0 to $1.5 \mathrm{~V}$ (vs. SCE), in phosphate buffer, $\mathrm{pH}=7.4$. Such ECP has been postulated to nanostructure the CFE surface, by increasing the density of defects and nanocracks on the surface, as a result of surface reactions of graphite, which occur during the treatment.

The surface structure of the nanostructured carbon fiber sensor (N-CFS) was examined using scanning electron microscope (SEM) [6]. SEM images of the cleaved polyacrylonitrile (PAN) carbon fibers surface show highly irregular nanofeatures formed during the manufacturing process, and visible defects and pores. After the electrochemical treatment, the pores and cracks along the transverse ridges are exposed and increase in width from 140 to $200 \mathrm{~nm}$ along the edge of the fiber, and nodules are visible in some regions of the surface. Thus, electrochemical fabrication of the CFS surface enhances the nanofeatures that are present at the surface of PAN fibers as a result of the manufacturing process [7].

The electrochemically treated CFS have a stable background current, which can be subtracted well from the current produced by 2,8-DHA, and this contributes to the increase in the sensitivity of the measurements of 2,8-DHA by fast scan voltammetry (FSV) [8]. FSV measurements require only several seconds to complete [9]. In addition, in FSV the $\mathrm{S} / \mathrm{N}$ ratio of the signal from the analyte $(2,8-\mathrm{DHA})$ to noise, from the electrochemical signal from the background current, is improved because of the high temporal resolution of FSV which allows acquisition of a large number of FSV cycles in a short period of time, which can be signal averaged. 
The rapid signal acquisition in FSV is an advantage for the determination of more difficult-to-measure biological analytes, such as 2,8-DHA. 2,8-DHA displays complex electrochemical response, allowing different chemical reactions to occur during its measurements. However, these reactions can be outrun at the fast scan rates allowed by FSV, thus improving selectivity and sensitivity and reducing passivation of the sensor [10]. Furthermore, the sensor surface can be regenerated during signal acquisition by FSV to prolong sensor lifetime [6,7]. Fabrication and characterization of the CFS have been described $[7,9]$. The use of FSV for measurements of the important metabolites adenosine and xanthine, which are difficult to analyze with standard methods and sensors, has been described $[7,9,10]$.

\section{Experimental}

\subsection{Chemicals}

All chemicals were of the analytical reagent grade and were used as received. All chemicals, except for HBSS buffer, were obtained from Fisher Scientific (Pittsburgh, PA) and SigmaAldrich (St. Louis, MO). Solutions were prepared in doubly deionized water daily before experiments. Phosphate buffer, $\mathrm{pH}=7.4$, contained sodium phosphate monobasic monohydrate $\left(\mathrm{NaH}_{2} \mathrm{PO}_{4} \cdot \mathrm{H}_{2} \mathrm{O}\right)$ and dibasic anhydrous $\left(\mathrm{Na}_{2} \mathrm{HPO}_{4}\right)$ at a total concentration of $31 \mathrm{mM}$. The $\mathrm{pH}$ was adjusted with 0.1 $\mathrm{M} \mathrm{NaOH}$ before experiments. HBSS (Hank's Balanced Salt Solution) $\left(\mathrm{CaCl}_{2} 1.26 \mathrm{mM}, \mathrm{MgCl}_{2} .6 \mathrm{H}_{2} \mathrm{O} 0.493 \mathrm{mM}, \mathrm{MgSO}_{4} .7 \mathrm{H}_{2} \mathrm{O}\right.$ $0.407 \mathrm{mM}, \mathrm{KCl} 5.33 \mathrm{mM}, \mathrm{KH}_{2} \mathrm{PO}_{4} 0.441 \mathrm{mM}, \mathrm{NaHCO}_{3} 4.17 \mathrm{mM}$, $\mathrm{NaCl} 137.93 \mathrm{mM}, \mathrm{Na}_{2} \mathrm{HPO}_{4} 0.338 \mathrm{mM}$, and D-glucose $5.56 \mathrm{mM}$ ) was obtained from Invitrogen (Carlsbad, CA). 2,8-DHA was prepared before the experiments in $31 \mathrm{mM}$ phosphate buffer and in HBSS: phosphate buffer $(1: 5)(\mathrm{pH}=7.4)$. All determinations were performed at room temperature. All measurements were repeated at least in triplicate. The results from determinations at different carbon fiber sensors were pooled. The reported results reflect the reproducibility of the measurements and the reproducibility of fabrication of carbon fiber sensors.

\subsection{Instrumentation}

A Bioanalytical Systems Electrochemical Analyzer (BAS100 , West Lafayette, IN) with preamplifier was used at scan rates $<1 \mathrm{~V} / \mathrm{s}$ [11]. For FSV in a two electrode system, a continuous triangular waveform was applied to the reference electrode with a function generator (Universal Programmer, model 175, EG\&G Princeton, NJ). The CFS was continuously cycled for 3600 cycles for $30 \mathrm{~min}$ between the potentials of -1.0 to $1.5 \mathrm{~V} v s$. SCE at $10 \mathrm{~V} / \mathrm{s}$. The background signal obtained in buffer by averaging 250 fast scan voltammograms in the potential window of -1.0 to $1.5 \mathrm{~V}$ was subtracted from 250 averaged fast scan voltammograms for each sample. The current was stored (LeCroy Oscilloscope 9310A, Chestnut Ridge, NY) and the data were transferred to a $\mathrm{PC}$ and processed using Origin 6.0 (Microcal Software, Inc., Northampton, MA).

Polyacrylonitrile-based (PAN) carbon fiber microdisks (7.5 mm diameter; Textron Specialty Materials, Lowell, MA) were used as the working electrodes. The sensor fabrication procedure has been described in detail $[7,9,10]$. The fabrication was modified by touch polishing of the sealed fiber on 600-grit SiC paper (Mark V Laboratory, East Granby, CT) on a polishing wheel instead of a 1 min polishing time (Ecomet I, Buehler Laboratory, Evanston, IL). The surface was washed with doubly distilled water before measurements. Sensors that gave a response in $5 \mathrm{mM} \mathrm{K}_{3}\left[\mathrm{Fe}(\mathrm{CN})_{6}\right]$ in $0.5 \mathrm{M} \mathrm{KCl}(\mathrm{pH}=6.0)$ at 50 $\mathrm{mV} / \mathrm{s}$ corresponding to the theoretical diameter of the carbon fiber $(7.5 \mathrm{~mm})$ were used for analysis. A disk geometry was assumed and the diameter calculated using the diffusion coefficient of $\mathrm{Fe}(\mathrm{CN})_{6^{3-}}=7.7 \times 10^{-6} \mathrm{~cm}^{2} \mathrm{~s}^{-1}$. Saturated calomel
(SCE) was the reference electrode (Fisher Scientific, Pittsburg, PA).

\subsection{Electrochemical measurements}

\subsubsection{Voltammetry of ferricyanide before and after ECP of CFS}

As mentioned above, voltammograms of $\mathrm{K}_{3}\left[\mathrm{Fe}(\mathrm{CN})_{6}\right]$ were used to evaluate the radius of carbon fiber sensors before and after the electrochemical pretreatment (ECP) in physiological buffer $\mathrm{pH}=7.4$ of the sensor surface. Figure 1 shows the voltammetric i-E curves of $\mathrm{Fe}(\mathrm{CN})_{6}{ }^{3-}$ that were obtained before (Figure 1a) and after (Figure $1 \mathrm{~b}$ and c) the electrochemical pretreatment (ECP) of the CFS in $31 \mathrm{mM}$ phosphate and in HBSS: phosphate buffers $(1: 5) \mathrm{pH}=7.4$ respectively. A 1:5 dilution improved the background subtraction relative to undiluted HBSS. In Figure 1 a visible change in the shape of the $i-E$ curve of $\mathrm{Fe}(\mathrm{CN})_{6}{ }^{3-}$ is apparent after the ECP of the CFS. Before the ECP, the shape of the current plateau is not welldefined and the current rises slowly, as shown in Figure 1a. After ECP, a well-defined steady-state plateau is observed with a steep current rise on the approach to the plateau (Figure 1b and $c)$. The change in shape of $i-E$ curves of ferricyanide at carbon fiber sensors after the ECP is a direct indication of changes at the CFS surface as a result of the pretreatment. The $i-E$ curves indicate that the CFS surface changes as a result of the ECP to more active surface formed as a result of the electrochemical pretreatment.

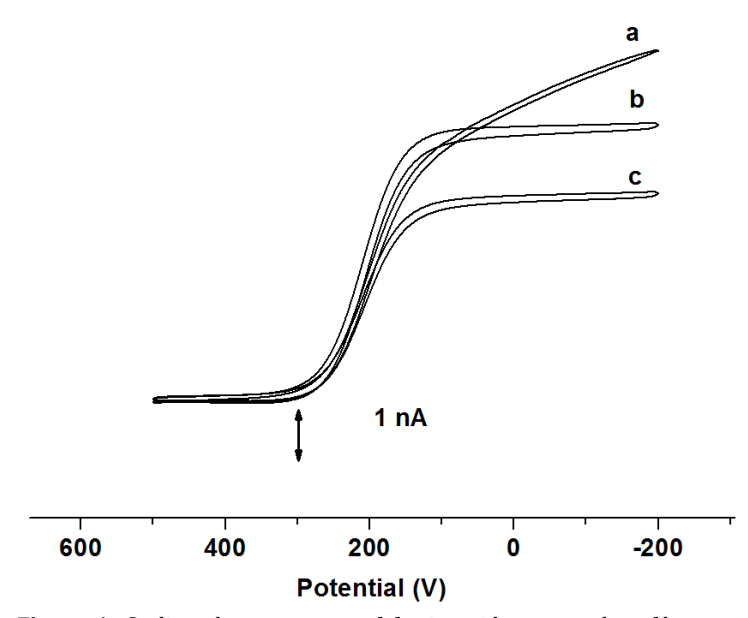

Figure 1. Cyclic voltammograms of ferricyanide at a carbon fiber sensor before (a) and after (b and c) the electrochemical pretreatment in $31 \mathrm{mM}$ phosphate and in 1:5 HBSS: phosphate buffers, respectively, $\mathrm{pH}=7.4 ; 5.0$ $\mathrm{mmol} / \mathrm{l} \mathrm{Fe}(\mathrm{CN})^{3+}, 0.5 \mathrm{M} \mathrm{KCI}, 50 \mathrm{mV} / \mathrm{s}, r=3.6 \pm 0.04 \mu \mathrm{m}$.

Higher activity of the CFS after the ECP in the electrode reaction of ferricyanide is confirmed from the analysis of the $i-E$ curves in Figure 1. The slope of the $E v s . \log \left(i_{l}-i\right) / i$ plot of the curves obtained for ferricyanide at $\mathrm{pH}=6.0$ decreases to $59 \pm 10$ $\mathrm{mV}$ after ECP from $98 \pm 10 \mathrm{mV}$ before ECP in $31 \mathrm{mM}$ phosphate buffer $\mathrm{pH}=7.4$ and from $114 \pm 10 \mathrm{mV}$ to $64 \pm 5$ in $1: 5$ HBSS: phosphate buffer $\mathrm{pH}=7.4$. After the ECP $E^{o^{\prime}}$ value shifted from $150 \pm 10 \mathrm{mV}$ to $187 \pm 10 \mathrm{mV}$ in phosphate and to $198 \pm 10 \mathrm{mV}$ in 1:5 HBSS: phosphate buffer. $E^{o^{\prime}}$ of $212 \mathrm{mV}$ (vs. SCE) has been reported for ferricyanide in $0.5 \mathrm{M} \mathrm{KCl} \mathrm{[12].} \mathrm{A} \mathrm{slope} \mathrm{of} 59 / \mathrm{n} \mathrm{mV}$ is expected for a reversible electrode reaction of ferricyanide [13].

After the ECP of the CFS the separation between the forward and reverse current traces in slow scan voltammograms of $\mathrm{Fe}(\mathrm{CN})_{6}{ }^{3-}$ increased at $-0.100 \mathrm{~V}$ in HBSS: phosphate buffer $(1: 5)($ ca. $0.13 \pm 0.03 \mathrm{nA})$ and in $31 \mathrm{mM}$ phosphate buffer $(c a .0 .12 \pm 0.02 \mathrm{nA})>$ before treatment $(c a$. $0.08 \pm 0.01$ ). 


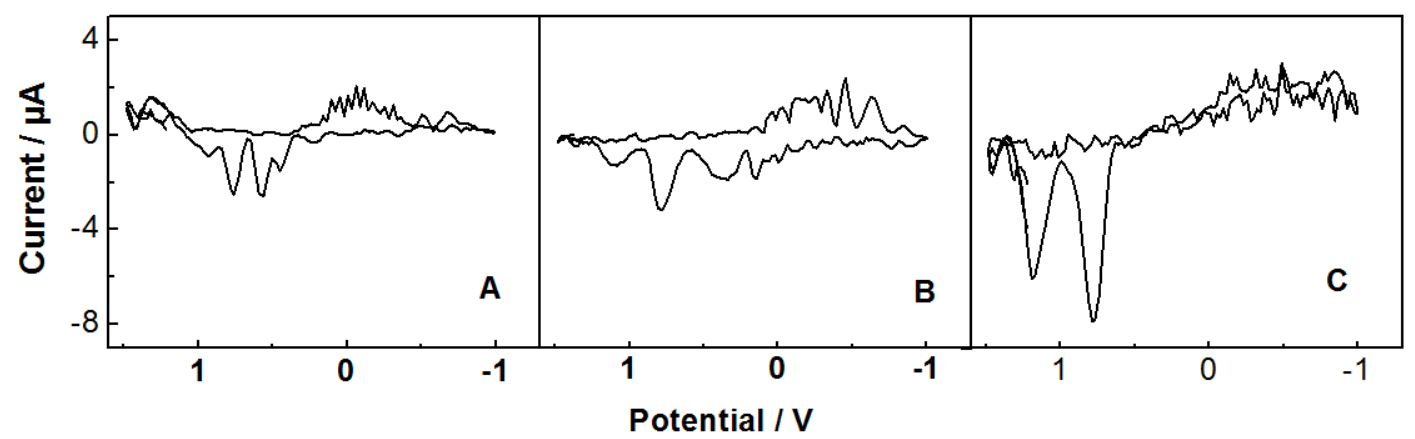

Figure 2. Fast scan cyclic voltammograms of $10 \mu \mathrm{M}$ 2,8-DHA at pH = 7.4 at $250 \mathrm{~V} / \mathrm{s} \mathrm{(A),} 500 \mathrm{~V} / \mathrm{s}$ (B) and $1000 \mathrm{~V} / \mathrm{s}$ (C) 1:5 HBSS: phosphate buffer, 250 cycles. Results after background subtraction $r=3.6 \pm 0.04 \mu \mathrm{m}$.

The increased separation between the forward and reverse current traces of ferricyanide (Figure 1) was best seen at the foothill and in the plateau region of the slow scan voltammetry $i-E$ curves [9]. The observed increase in the separation of the forward and reverse current traces in slow scan voltammetry $i$ $E$ curves reflects an increase in CFS background current. The increase in the background current in turn indicates an increase in CFS area and capacitance after the ECP of the CFS surface [14].

\subsubsection{Background current and the electrode capacitance}

The formation of nanocracks and edge nanostructures at the surface of carbon fiber sensor, to yield a highly active surface for the determination of purines, has been studied [15].

The background current observed at CFS in fast scan voltammetry, in a blank solution, is due to several processes including double-layer charging, redox reactions of the surface functional groups, such as quinones, and the redox reactions of the solution impurities that may be present in buffer.

The amplitude of the background current may be more than two orders of magnitude greater than that of the oxidation current of an analyte [16]. Consequently, FSV requires a stable background current to produce a reliable background that can be used in background subtraction. The formation of oxides [17] and the roughness of the sensor surface can change during a voltammetric determination and these changes can produce significant modifications of the background current [16].

In previous work, it was established that a stable background current can be obtained at a CFS after $30 \mathrm{~min}$ of continuous cycling of the electrode potential in a potential window from -1.0 to $1.5 \mathrm{~V}$ ( vs. SCE), in $31 \mathrm{mM}$ phosphate buffer at $\mathrm{pH}=7.4$, at a scan rate of $10 \mathrm{~V} / \mathrm{s}$ [9]. The relatively low scan rate and a positive potential limit of the electrochemical pretreatment process was shown to contribute to high background current which reflects a high electrode capacitance of the CFS. The CFS capacitance after the ECP was shown to increase with an increase in a positive potential limit of the pretreatment $[15,16]$. The increase in the capacitance reflects an increase in the electrode surface area following the electrochemical pretreatment of the surface. The apparent increase in the capacitance that has been reported after ECP is in agreement with the results in Figure 1. It has been shown that when the negative potential limit of the ECP is set at $-1.0 \mathrm{~V}$, the charging current and the electrode capacitance is high, especially when the positive potential limit of the pretreatment is set at $1.5 \mathrm{~V}[15,16]$. The high charging current and the high electrode capacitance have been related to the formation of surface nanocracks as a result of surface reactions which can occur during the electrochemical pretreatment [9].

\subsubsection{Determination of the electrode capacitance}

The apparent electrode capacitance before and after the ECP, Cobs $\left(\mu \mathrm{F} \mathrm{cm}^{-2}\right)$, was measured at $0.75 \mathrm{~V}$ (vs. SCE). It was assumed that at $0.75 \mathrm{~V}$ the current resulting from surface faradaic reactions is low and the current which is measured at these potential results predominantly from double layer charging [18].

The equation which was used to calculate the capacitance from the experimental background current is $\mathrm{C}_{\mathrm{obs}}=\mathrm{I} / 2 v \mathrm{~A}$. The observed capacitance is normalized by the electrode area, A $\left(\mathrm{cm}^{2}\right)$, that was obtained from the electrode radius determined from the limiting current of $\mathrm{Fe}(\mathrm{CN})_{6}{ }^{3-}$. In the above equation, $I$ (A) is the background current which is measured at $0.75 \mathrm{~V}$ and $v$ is the scan rate $(\mathrm{V} / \mathrm{s})$. The background (capacitive) current was measured in $\mathrm{pH}=7.4$ phosphate buffer and in HBSS: phosphate buffer (1:5) supporting electrolytes alone from the anodic and the cathodic currents [15]. The background current was recorded in the electrolyte solution in the absence of an analyte before and after the ECP. As reported previously [16] ECP increases the background current in the entire potential window. Typical capacitance values that were determined before and after the ECP were $650 \pm 150,1100 \pm 250 \mu \mathrm{F} / \mathrm{cm}^{2}$ in phosphate buffer and $1118 \pm 100,2029 \pm 150 \mu \mathrm{F} / \mathrm{cm}^{2}$ in HBSS:phosphate buffer (1:5), respectively [15].

As shown from capacitance values, CFS background current in $\mathrm{pH}=7.4$ HBSS:phosphate buffer (1:5) is higher than in $\mathrm{pH}=$ 7.4 phosphate buffer in the entire potential window. At positive potentials of $c a .1 .5 \mathrm{~V}$ high background current at CFS in 1:5 HBSS: phosphate is due in part to chlorides in the buffers and the ability of chloride ions or chlorine, which can evolve at positive potentials, to penetrate the electrode surface $[19,20]$. CFS background peak at $c a .0 .0 \mathrm{~V}$ has been associated with surface functional groups such as the quinone $[17,19]$.

The potential window was selected to facilitate the electrochemical reactions at the surface, surface reactions, which produce and maintain the CFS surface structure. As a result of these reactions CFS surface is stable (as reflected in the stable background current) and sensitive in the determinations of 2,8-DHA by FSV in the same potential window.

\section{Results and discussion}

\subsection{2,8-DHA at different scan rates}

2,8-DHA at CFS was investigated in phosphate, and HBSS:phosphate physiological buffers $(1: 5) \mathrm{pH}=7.4$ by FSV. 


\begin{tabular}{|c|c|c|c|c|c|}
\hline Scan Rate (V/s) & Peak Potential (vs SCE) & Sensitivity $(\mathrm{nA} / \mu \mathrm{M})$ & LOD $(\mu \mathrm{M})$ & LDR $(\mu \mathrm{M})$ & $r$ \\
\hline $250^{a}$ & $\begin{array}{l}0.56 \\
0.76\end{array}$ & $\begin{array}{l}0.26 \pm 0.01 \\
0.25 \pm 0.02\end{array}$ & 2 & $2-20$ & $0.996(n=6)$ \\
\hline 500 b & 0.76 & $0.40 \pm 0.01$ & 1 & $1-20$ & $0.998(\mathrm{n}=6)$ \\
\hline $1000^{\text {a }}$ & $\begin{array}{l}0.76 \\
1.14 \\
\end{array}$ & $\begin{array}{l}0.31 \pm 0.01 \\
0.24 \pm 0.02 \\
\end{array}$ & 2 & $2-20$ & $0.996(\mathrm{n}=6)$ \\
\hline
\end{tabular}

a Sensitivity was determined for $10 \mu \mathrm{M}$ 2,8-DHA.

b Sensitivity was calculated from a calibration plot, concentration range 1-20 $\mu \mathrm{M}$.
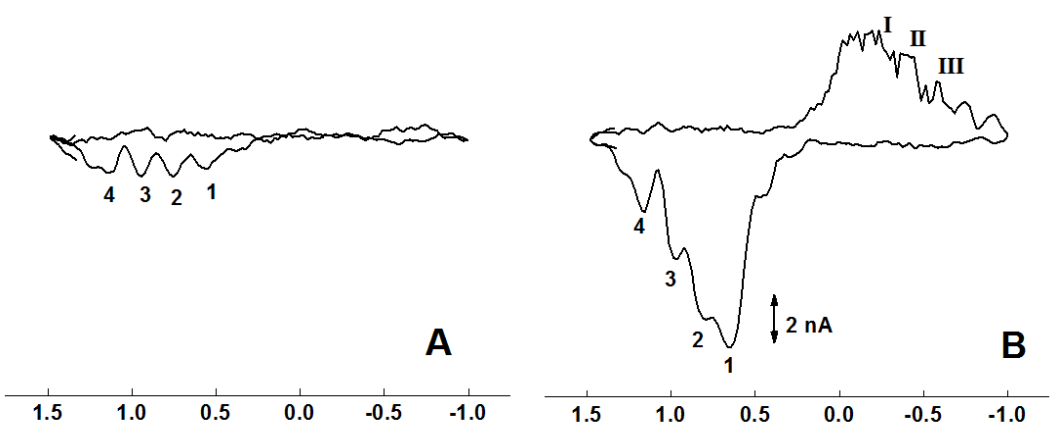

\section{Potential (V)}

Figure 3. Fast scan cyclic voltammograms of (A) $1 \mu \mathrm{M}$ and (B) $25 \mu \mathrm{M}$ 2,8-DHA in 1:5 HBSS : phosphate buffer, $\mathrm{pH}=7.4$. Scan rate $500 \mathrm{~V} / \mathrm{s}$, electrode radius $=$ $3.6 \pm 0.04 \mu \mathrm{m}$.

2,8-DHA was measured at $31 \mathrm{mM}$ phosphate buffer $\mathrm{pH}=$ 7.4 and in 1:5 HBSS: Phosphate buffer because of the previously reported high sensitivity of FSV with the CFS in these buffers by our group [6,7].

Figure 2 shows typical voltammograms of 2,8-DHA that were obtained by FSV at carbon fiber sensors after the electrochemical pretreatment of the sensor. The voltammograms were obtained for low concentrations of 2,8DHA $(10 \mu \mathrm{M})$ in HBSS:phosphate buffer $(1: 5) \mathrm{pH}=7.4$ at a voltammetric scan rate of 250 (A), 500 (B) and 1000 (C) V/s. The results shown in Figure 2 show the FSV results after background subtraction. The voltammograms were recorded in the potential window from -1.0 to $1.5 \mathrm{~V}$ (vs. SCE) which is the same as the potential window which was used in the ECP of the CFS. Before each measurement the same potential window was used to record the background current which was used in background subtraction.

As shown in Figure 2, at scan rates of $250 \mathrm{~V} / \mathrm{s}, 2,8-\mathrm{DHA}$ shows two well-developed peaks at a potential of ca. $0.70 \pm 0.06$ $\mathrm{V}, 0.55 \pm 0.05 \mathrm{~V}$ and two shoulders at a potential of $c a .0 .45 \pm 0.04$ and $0.95 \pm 0.06 \mathrm{~V}$ (vs. SCE). Two small reduction peaks were also observed at $-0.58 \pm 0.03$ and $-0.62 \pm 0.04 \mathrm{~V}$, respectively. At higher scan rates $(500 \mathrm{~V} / \mathrm{s})$, the intensity of the oxidation peak appears at $0.55 \mathrm{~V}$ shifted to less positive potential ca. $0.32 \mathrm{~V}$ (Figure 2B) and disappeared at scan rate of $1000 \mathrm{~V} / \mathrm{s}$ (Figure 2C). The intensity of the oxidation peak appears at $c a$. $0.70 \pm 0.06 \mathrm{~V}$ increases with increasing scan rate. Also three reduction peaks were observed at $-0.45 \mathrm{~V},-0.63 \mathrm{~V}$ and $-0.84 \mathrm{~V}$, respectively. At lower scan rates $(250 \mathrm{~V} / \mathrm{s})$ the intensity of the oxidation peak appeared as shoulder at ca. $0.93 \mathrm{~V}$ (Figure 2A) increases at higher scan rates of 500 and $1000 \mathrm{~V} / \mathrm{s}$ and shifted to more positive potential of $c a .1 .12 \mathrm{~V}$ (Figure 2B and 2C, respectively). The increase and decease in the intensity of the oxidation peaks may be attributed to the proposed oxidation pathway of 2,8-DHA [21,22].

The peak potentials of 2,8-DHA as well as the sensitivity and LOD of 2,8-DHA that were determined at different scan rates are summarized in Table 1.

The behavior of 2,8-DHA in FSV at CFS at $500 \mathrm{~V} / \mathrm{s}$ is shown in Figure 3 for $1 \mu \mathrm{M}$ and $25 \mu \mathrm{M}$, respectively. The oxidation process at $0.65 \pm 0.07 \mathrm{~V}$ (peak 1) was proposed to be due to the formation of a diimine by a $2 \mathrm{e}^{-}, 2 \mathrm{H}^{+}$oxidation of 2,8-DHA. Fast scan voltammetry at the CFS has better LOD $(1 \mu \mathrm{M})$ than reported previously for 2,8-DHA [21]. Furthermore it allows the resolution of the complex oxidation processes attributed to 2,8-DHA dimer formation and subsequent oxidation [21,22,23]. It has been suggested that the dimers should be detected at high scan rates [21]. The three small reduction peaks observed at cyclic voltammograms of 2,8-DHA (I, III and III) may correspond to the reduction of the oxidation products formed in the reactions at $0.70 \pm 0.06$ (2) and $1.10 \pm 0.02$ (4) $\mathrm{V}$, respectively (Figure 3).

High sensitivity allows the detection of the peaks at $\mathrm{pH}=$ 7.4, which is otherwise difficult [21]. Peak potentials for the forward and reverse processes are close, suggesting adsorption of 2,8-DHA.

Scheme 1 shows the proposed oxidation and dimer formation pathway of 2,8-DHA. According to studies of adenine oxidation it can be concluded that the first $2 \mathrm{e}^{-}, 2 \mathrm{H}^{+}$oxidation of adenine (1) will lead to 2-hydroxyadenine (2) which on further $2 \mathrm{e}^{-}, 2 \mathrm{H}^{+}$oxidation will form 2,8-dihydroxyadenine $(3)$ as is reported for adenine in the literature [24]. 2,8-DHA undergoes further oxidation involving a $2 \mathrm{e}^{-}, 2 \mathrm{H}^{+}$step to give the corresponding diimine (4).

At physiological $\mathrm{pH}$ only an 0-0 dimer was formed. The formation of dimer with an 0-0 linkage can be explained on the basis of a $1 \mathrm{e}^{-}, 1 \mathrm{H}^{+}$oxidation of 2,8-dihydroxyadenine (3). Oxidation of 8-hydroxypurines in a $1 \mathrm{e}^{-}, 1 \mathrm{H}^{+}$step to form a variety of dimers has been reported previously $[25,26]$. It appears that 2,8-DHA undergoes $1 \mathrm{e}, 1 \mathrm{H}^{+}$oxidation to give a radical species (5) which rapidly dimerizes with another similar moiety to form dimer (6) as shown in Scheme 1.

\subsection{Sensitivity of 2,8-DHA at pretreated CFS}

Table 1 shows that 2,8-DHA can be determined with high sensitivity by FSV at carbon fiber senor after the ECP. Peak 1 at ca. $0.70 \pm 0.06 \mathrm{~V}$ was chosen for 2,8-DHA measurements, because the peak is well developed in the expected (biological) concentration range. By increasing the scan rate, 2,8-DHA peak current increases. The increase in the 2,8-DHA current is accompanied by the increase in background current. 


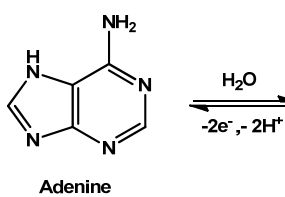

(1)

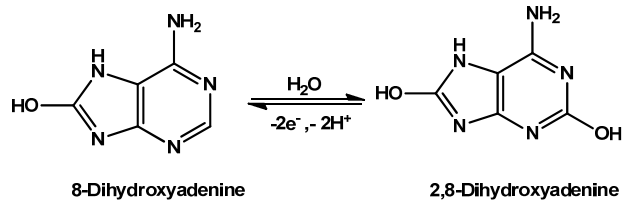

(2)

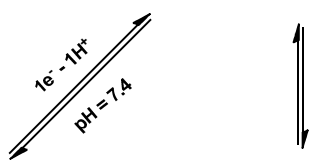<smiles>Nc1nc(O)nc2[nH]c([O-])nc12</smiles>

(5)

Radical<smiles>Nc1nc(O)cc2[nH]c(OOc3nc4c(N)nc(O)nc4[nH]3)nc12</smiles><smiles>Nc1nc(O)cc2[nH]c(OOc3nc4c(N)nc(O)nc4[nH]3)nc12</smiles>

(6)
(3)<smiles>NC1=NC(=O)N=C2NC(=O)N=C12</smiles>

(4)

Diimine

Dimer

Scheme 1

Consequently, after background subtraction the best sensitivity and signal-to-noise ratio for 2,8-DHA determinations is obtained at scan rates of $500 \mathrm{~V} / \mathrm{s}$ (Table 1).

The sensitivity for 2,8-DHA by FSV was found at $500 \mathrm{~V} / \mathrm{s}$ to be $0.40 \pm 0.01 \mathrm{nA} / \mu \mathrm{M}\left(r^{2}=0.998\right)$ from the calibration plot of 2,8- DHA, with a linear dynamic range from 1 to $20 \mu \mathrm{M}$, and a limit of detection of $1 \mu \mathrm{M}$ as shown in Figure 4.

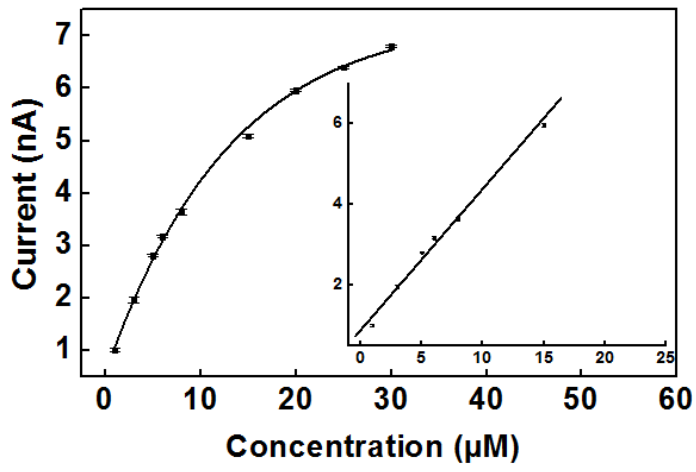

Figure 4. A calibration plot for sensitivity determination of 2,8-DHA; linear dynamic range $(1-20 \mu \mathrm{M})$; LOD $1 \mu \mathrm{M}$; 1:5 HBSS: Phosphate buffer $\mathrm{pH}=7.4$, scan rate $500 \mathrm{~V} / \mathrm{s}$.

Based on the calibration curve sensitivity for $1 \mu \mathrm{M}$ 2,8-DHA, the expected peak (1) current is $0.36 \mathrm{nA}$, but the peak is not observed. However, a well-developed peak is seen at more positive potentials (Figure 3). For $25 \mu \mathrm{M}$ 2,8-DHA, peak (2) $(0.70 \pm 0.06 \mathrm{~V})$ is a shoulder on the broad oxidation peak, and the peak current, $5.6 \pm 0.3 \mathrm{nA}$, is lower than expected based on the calibration curve sensitivity (1-20 $\mu \mathrm{M}$ concentration range).

\section{Conclusion}

The method of carbon fiber Sensor surface activation, which was used here, produces a nanostructured carbon fiber surface, with defects, and cation exchanged surface oxides. The activation method that was used produces a CFS surface, which is sensitive in 2,8-dihydroxyadenine determinations under physiological conditions. The apparent fast electrode kinetics of 2,8-DHA, at the carbon fiber sensor that was produced, contributes to the high sensitivity in 2,8-DHA determinations by FSV. High sensitivity of the new method of 2,8-DHA determinations that is shown here, will facilitate the use of CFS with FSV technique, in the determination of 2,8-DHA in vivo, for the early detection of kidneys failure as a result of adenine phosphoribosyltransferase enzyme deficiency which lead to the precipitation and crystallization of 2,8-DHA and stone formation in various parts of the urinary tract, leading to the loss of the kidneys.

\section{Acknowledgments}

This work was funded by Suez Canal University under the supervision of Egyptian Higher Education Ministry. I would like to sincerely thank Dr. Anna Brajter-Toth for her cooperation in using her research laboratory at the Department of Chemistry, University of Florida to do this work, and also for her helpful discussion. I would also like to thank Dr. Mehjabin Kathiwala and Rachel Cohen-Shohet from University of Florida, 
Department of Chemistry for help with sensors preparation and activation process.

\section{References}

[1]. Wessel, T.; Lanvers, C.; Fruend, S.; Hempel, G. J. Chromatogr. A 2000, 894, 157-164.

[2]. McCaskey, P. C.; Rigsby, W. E.; Hinton, D. M.; Friedlander, L.; Hurst, V. J. Vet. Pathol. 1991, 28, 99-109.

[3]. Deng, L.; Yang, M.; Fründ, S.; Wessel, T.; De Abreu, R. A.; Tischfield, J. A.; Sahota, J. A. Mol. Genet. Metab. 2001, 72, 260-264.

[4]. Jacomelli, G.; Micheli, V.; Peruzzi, L.; Notarantonio, L.; Cerboni, B.; Sestini, S.; Pompucci, G. Clin. Chim. Acta 2002, 324, 135-139.

[5]. Safranow, K.; Machoy, Z. J. Chromatogr. B Analyt. Technol. Biomed. Life Sci. 2005, 819(2), 229-235.

[6]. Kathiwala, M.; Abou El-Nour, K. M.; Cohen-Shohet, R.; Brajter-Toth, A. Analyst 2010, 135, 296-301.

[7]. Kathiwala, M.; Affum, A. O.; Perry, J.; Brajter-Toth, A. Analyst 2008, 133, 810-816.

[8]. Bravo, R.; Hsueh, C. C.; Jamarillo, A.; Brajter-Toth, A. Analyst 1998, 123, 1625-1630.

[9]. Brajter-Toth, A.; Abou El-Nour, K.; Cavalheiro, E. T.; Bravo, R. Anal. Chem. 2000, 72, 1576-1584.

[10]. Abou El-Nour, K.; Brajter-Toth, A. Electroanalysis 2000, 12, 805-810.

[11]. Hsueh, C. C.; Brajter-Toth, A. Anal. Chim. Acta 1996, 321, 209-214.

[12]. Bard, A. J. The Encyclopedia of The electrochemistry of The Elements, Marcel Dekker, 1973.

[13]. Bard, A. J.; Faulkner, R. L. Elechochemical Methods: Fundamentals and Application, Wiley, 1980.

[14]. Green, S. J.; Stokes, J. J.; Hostetler, M. J.; Pietron, J.; Murray, R. W. J. Phys. Chem. B.1997, 101(14), 2663-2668.

[15]. Bravo, R.; Brajter-Toth, A. Chem. Anal. (Warsaw) 1999, 44, 423-436.

[16]. Hsueh, C. C.; Bravo, R.; Jaramillo, A.; Brajter-Toth, A. Anal. Chim. Acta 1997, 349, 67-76.

[17]. McCreery, L. R. Electroanalytical Chemishy, in: Bard, A. J. (Ed.), Marcel Dekker, 1991, pp. 221-225.

[18]. Anjo, D. M.; Kahr, M.; Khodabakhsh, M. M.; Nowinski, S.; Wagner, M. Anal. Chem. 1989, 61, 2603-2608.

[19]. Kinoshita, K. Carbon: Electrochemical and Physical Properties, Wiley \& Son, 1988.

[20]. Falat, L.; Cheng, H. Y. J. Electroanal. Chem. 1983, 157, 393-397.

[21]. Diculescu, V. C.; Piedade, J. A. P.; Oliveira-Brett, A. M. Bioelechemistry 2007, 70, 141-146.

[22]. Goyal, R. N.; Mittal, A. Indian J. Chem. 1993, 32A, 852-856.

[23]. Goyal, R. N.; Sangal, A. J. Electroanal. Chem. 2003, 557, 147-155.

[24]. Goyal, R. N.; Sangal, A. J. Electroanal. Chem. 2002, 521, 72-80.

[25]. Subramanian, P.; Dryhurst, G. J. Electroanal. Chem. 1987, 224, 137 162.

[26]. Oliveira-Brett, A. M.; Diculescu, V. C.; Piedade, J. A. P. Bioelechemistry 2002, 55, 61-62. 